\section{Educación y territorio: abordajes desde el Programa Integral Metropolitano de la Universidad de la República}

\author{
Agustín Cano Menoni \\ acano@pim.edu.uy \\ (iD) orcid.org/0000-0001-6076-5580 \\ Valeria Cavalli Dalla Rizza \\ vcavalli@psico.edu.uy \\ (iD) orcid.org/0000-0002-9006-2242 \\ Carina Alejandra Cassanello \\ carinacassanello@gmail.com \\ (iD) orcid.org/0000-0002-0649-4799
}

RECEPCIÓN: 27/06/19

ACEPTACIÓN FINAL: 09/09/19

\section{Resumen}

El siguiente artículo reflexiona sobre las características y potencialidades de abordar las políticas educativas y los procesos pedagógicos desde el territorio y un enfoque de diálogo de saberes, en el marco de un programa integral de la Universidad de la República (UdelaR) que articula las funciones universitarias de extensión, investigación y enseñanza. Para ello, presentaremos al Programa Integral Metropolitano (PIM) y tomaremos específicamente las líneas de trabajo que lleva adelante el Núcleo de intervención e investigación Educación y Territorio de dicho Programa. En ese sentido, se desarrollará el marco histórico, teórico y metodológico que define su quehacer, las especificidades del territorio de incidencia donde lleva adelante sus actividades y las líneas de investigación que el núcleo desarrolla en torno a temáticas y problemáticas educativas emergentes de los procesos de extensión y articulación con las instituciones educativas y organizaciones sociales de la zona.

Palabras clave: educación, territorio, integralidad, saberes, alternativas pedagógicas.

\author{
Juan Caggiani Gómez \\ juancaggiani@gmail.com \\ (iD) orcid.org/0000-0003-1799-7225 \\ Programa Integral Metropolitano de la \\ Universidad de la República, Uruguay.
}

Education and territory: approaches from the Metropolitan Integral Program of the University of the Republic

\section{Abstract}

This article reflects on the characteristics and potentialities of addressing the educational policies and the pedagogical processes from a territorial approach, within the framework of an integral program of the Universidad de la República de Uruguay (UdelaR) that develops the university functions of extension, research and teaching. First, we present the Programa Integral Metropolitano (PIM), focusing on the historical, theoretical and methodological framework that defines the profile and work of this type of university programs. Secondly, we present some socio-economic and educational characteristics of the program's territory. Finally, we focus on the lines of work carried out by the intervention and research nucleus in Education and Territory, presenting the research lines and the social intervention strategies.

Keywords: education, territory, integrality, knowledge, pedagogical alternatives.
Cultura(s) en clave de extensión universitaria / Desafíos de gestión 다(1)(2)(2)
Educação e território: abordagens desde o Programa Integral Metropolitano da Universidade da República

\section{Resumo}

Este artigo reflete sobre as características e potencialidades de abordar as políticas educacionais e os processos pedagógicos a partir do território e uma abordagem de diálogo de saberes, no âmbito de um programa abrangente da Universidade da República (UdelaR) que articula as funções universitárias de extensão, pesquisa e ensino. Para fazer isso, vamos apresentar o Programa Metropolitano Integral (PIM-UdelaR), abordando especificamente as linhas de trabalho que estão sendo realizadas pelo grupo de intervenção e pesquisa do Programa: Educação e Território. Nesse sentido, serão desenvolvidas as referências históricas, teóricas e metodológicas que definem o trabalho do PIM, as especificidades do território de incidência em que desenvolve as suas atividades, e as linhas de pesquisa que o grupo desenvolve em torno de problemas temáticos e educacionais emergentes dos processos de extensão e articulação com as instituições educacionais e organizações sociais da área.

Palavras-chave: educação, território, integralidade, saberes, alternativas pedagógicas.

Para citación de este artículo: Cano Menoni, A.; Cavalli Dalla Rizza, V.; Cassanello, C. y Caggiani Gómez, J. (2019). Educación y territorio: abordajes desde el Programa Integral Metropolitano de la Universidad de la República. +E: Revista de Extensión Universitaria, 9(11), 53-68. doi: 10.14409/ extension.v9i11.Jul-Dic.8678. 


\section{Introducción}

El Programa Integral Metropolitano (PIM) pertenece a la Universidad de la República (UdelaR) y comenzó a desarrollarse en 2008. Fue creado con el propósito de contribuir a la generación y fortalecimiento de programas académicos que se sustenten en el aprendizaje basado en problemas emergentes de la realidad con la participación de los actores sociales. A ese respecto, conjuga saberes, disciplinas y funciones universitarias para aportar a la creación de conocimiento y contribuir a buscar alternativas que logren una mejor calidad de vida de la comunidad involucrada. Complementariamente, pretende contribuir al establecimiento de vínculos sólidos entre la Universidad y la comunidad, con una mayor apropiación del espacio universitario por parte de esta a través de sus organizaciones comunales, barriales o sindicales.

El proceso de gestación, institucionalización y consolidación del PIM se enmarca en una discusión dentro de la UdelaR, interpelada por otros actores sociales, sobre el rol de la extensión universitaria en dos dimensiones complementarias: la formación integral y activa de los/as estudiantes universitarios y la creación de conocimiento mediante la incorporación de diferentes actores sociales y comunidades de pertenencia que la nutran con su realidad.

Por eso la intención del siguiente artículo es reflexionar sobre las características y potencialidades de abordar el "campo problemático de la educación" (Puiggrós, 1990) desde la perspectiva territorial y el enfoque de la extensión integral y el diálogo de saberes, en el marco de un programa universitario con las particularidades del PIM.

En un primer momento, desarrollaremos las características del PIM en relación con su contexto histórico, su construcción teórica y metodológica y sus formas organizativas. Asimismo, intentaremos esbozar lo que entendemos por extensión crítica, integralidad, diálogo de saberes y las especificidades de los Espacios de Formación Integral (EFI) como dispositivos centrales para la curricularización de la extensión. Planteamos también algunos datos cuantitativos socioeducativos que nos ayuden a entender qué características territoriales tiene el espacio geográfico donde se instala y desarrolla sus actividades el Programa, pensando que son estas especificidades las que van trazando múltiples territorialidades que necesariamente interpelan a los docentes del PIM, mostrándonos la gran fragmentación territorial y social que entra en escena.

Finalmente, presentaremos las líneas de investigación e intervención que el núcleo de educación viene desarrollando en función del trabajo en territorio y algunos ejemplos de proyectos conjuntos que se han llevado adelante con la comunidad para potenciar los procesos de generación de conocimiento en torno a diferentes problemáticas educativas.

\section{EI PIM, el enfoque territorial y los EFI}

Un programa universitario territorial de las características del PIM presenta peculiaridades organizativas, epistemológicas y pedagógicas. Por una parte, se dan las características que Rinesi (2015) identifica para lo que define como "universidades situadas", a las cuales se les plantea el desafío de, por una parte, realizarse plenamente en tanto universitas (es decir, desde una lógica de lo universal propia de los objetos universitarios) y, por otra parte, sostener la lógica situacional en diálogo con las organizaciones y comunidades de su entorno. La búsqueda por crear y afianzar procesos de complementariedad entre los dos ámbitos de sentido, esto es, el universitario y el territorial, implica generar procesos de carácter pedagó- 
gico entre la universidad y la sociedad y fortalecer un pensamiento crítico y emancipador. Al mismo tiempo, demanda la necesidad de promover la gestión participativa del Programa y el diálogo de los saberes académicos y populares.

Es preciso mencionar que el surgimiento del PIM se dio en un contexto político y académico favorable en la UdelaR para el desarrollo y consolidación de propuestas integrales. Este momento fue denominado como "Segunda Reforma Universitaria", lo cual evocaba la genealogía de la Universidad Latinoamericana heredera de la Reforma Universitaria de Córdoba. En este proceso, discusiones, prácticas y propuestas que se venían desarrollando a partir de experiencias extensionistas tomaron impulso para nutrir las discusiones de política universitaria en varias dimensiones. Una de ellas fue la curricularización de la extensión y renovación de la enseñanza, que permitió, entre otras cosas, otorgar institucionalidad a la integralidad, aprobar orientaciones para lo que se considera como procesos y actividades de extensión, e impulsar la incorporación de la extensión en clave integral en la formación universitaria (Tommasino et al., 2010).

Consecuentemente, en el marco de la misma discusión, se crearon los EFI como dispositivos de enseñanza orientados a promover la formación integral de los estudiantes universitarios. Estos implican, desde su definición, la integración de las tres funciones universitarias, de las disciplinas y de los saberes académicos y populares en una relación dialógica (Udelar, 2010). Esta integración, a su vez, se concebía como

"un proceso de avance paulatino y creciente (...). De este modo, se entiende que el peso de la enseñanza activa, de la investigación y la extensión puedan ser en un principio no del todo equilibrados, pero teniendo como meta un proceso superador que tienda hacia la integración y que genere una integración armónica y simultánea de las funciones”. (Udelar:19)

Así, los EFI como ámbitos de encuentro "activo" de saberes disciplinarios ante una realidad territorial compleja, heterogénea y contradictoria, requieren provocar y desafiar la construcción de una totalidad desde esas múltiples formas de aprehender la realidad y de interpretarla, de manera de articular las visiones distintas, potenciar la subjetividad como factor de conocimiento, integrar los diversos abordajes y avanzar hacia una construcción colectiva integral.

En 2009 se comenzaron a desarrollar EFI en diferentes servicios universitarios conforme a las características particulares de la formación que cada uno ofrece. Poco a poco fueron surgiendo propuestas intercarreras o interservicios, o aquellas que se proponen desde un servicio pero que son abiertas a la participación de estudiantes de otras disciplinas. Asimismo, se desarrollaron tanto EFI de sensibilización como de profundización. Los primeros se caracterizan por tener como objetivo el acercamiento de estudiantes a propuestas integrales, así como a sus referencias teóricas y metodológicas. Estos generalmente se implementan o son dirigidos a estudiantes en etapas de inicio de su formación universitaria. Por su parte, los EFI de profundización apuestan a desarrollo activo de prácticas integrales por parte de los estudiantes y usualmente están dirigidos a estudiantes avanzados. ${ }^{1}$

Las experiencias desarrolladas en el marco de los EFI, sobre todo aquellas que corresponden a la actividad extensionista, además de articular actores de diferentes servicios y 
disciplinas (actores universitarios), tienen que articular y ponerse en diálogo con actores no universitarios. En este sentido, podemos ver que las prácticas de estos espacios implican una salida al aula tradicional, una ruptura, para habilitar los espacios comunitarios y sociales como sitios de aprendizaje. Esto produce otros espacios, otros tiempos y otros actores. Esta condición que para nosotros es de gran riqueza también otorga una gran complejidad a la implementación de los EFI, en tanto es necesario contar con otros tiempos y otra presencia en territorios que cotidianamente los universitarios no habitan. Es allí donde los llamados "programa plataforma" ${ }^{2}$ cobran especial sentido al oficiar como espacios de articulación, acogida y sostén de propuestas universitarias en el territorio, lo cual les es permitido por su presencia cotidiana en los barrios y en conjunto con vecinos, vecinas, organizaciones e instituciones. Esta es, en parte, la función de los "programas integrales" como el PIM.

El origen del PIM se remonta al año 2007, cuando, a partir de la experiencia de un programa integral antecesor -el Programa APEX-Cerro ${ }^{3}$-, un grupo de trabajo elaboró los documentos que establecieron las bases conceptuales y metodológicas de la noción de "integralidad". ${ }^{4} Y$ en 2008 comenzó la etapa de implementación del Programa con un equipo docente inicial caracterizado por su interdisciplinariedad. Se organizaron cuatro equipos de trabajo que realizaron el diseño de una estrategia de acercamiento a los territorios para el conocimiento de su historia, de sus formas organizativas y de las necesidades y problemas emergentes. En 2010 se inició un tercer momento en la historia del Programa caracterizado por su reestructuración y mayor institucionalización, en cuyo marco se concretó la Casa del PIM en el territorio de incidencia. El mismo contexto de la UdelaR que favoreció la creación de los EFI coincidió para la ampliación de las actividades del PIM y la consolidación de su vínculo con el territorio, todo lo cual hizo necesaria una nueva organización académica complementaria a los Equipos Territoriales que adoptó el nombre de Ejes Temáticos. Los mismos agrupaban a los docentes de los distintos Equipos Territoriales en 4 áreas de conocimiento y acumulación académica del PIM: educación, trabajo, territorio y salud. También se diseñó una planificación estratégica que contempló tres dimensiones: una pedagógica, una política y otra referida a la gestión participativa. En 2015 empezó el proceso de consolidación del Programa. La organización de los equipos territoriales permitió el vínculo con los actores sociales y el territorio, base sobre la cual se articularon las prácticas universitarias de enseñanza e investigación, los EFI de profundización y de sensibilización y otras propuestas curriculares. Asimismo, en 2016 los ejes temáticos se convirtieron en Núcleos de Intervención e Investigación definidos como nueva forma organizativa orientada a la

2) "Programa plataforma" es una denominación que hace énfasis en la función de propiciar y articular la participación de las diferentes facultades y escuelas de la universidad en procesos de extensión, investigación y enseñanza en el territorio. "Plataforma" refiere entonces a ser un lugar donde los servicios pueden hacer extensión, de modo tal que, al retirarse, el Programa sostiene los vínculos con la comunidad, evitando el efecto "abandono" que en otras ocasiones se genera.

3) APEX es un acrónimo que hace alusión a aprendizaje-extensión. Se trata de un programa interdisciplinario integral de proyección comunitaria de la UdelaR, antecedente del PIM, que funciona desde 1993 en la zona de referencia del barrio Cerro y sus aledaños.

4) "Programa Integral Metropolitano: Hacia un país de aprendizaje, democracia e inclusión", documento elaborado por el grupo de trabajo creado por las comisiones sectoriales de Extensión, Investigación y Enseñanza, el Programa APEX-Cerro y la Federación de Estudiantes Universitarios del Uruguay en 2007 con el fin de concretar la creación del PIM. Dicho documento fue publicado en Programa Integral Metropolitano (2008:45-49). 
producción de conocimiento en las áreas temáticas sobre las que se había generado una acumulación de trabajos y abordajes.

Esto redundó en el afianzamiento de campos de conocimiento relativos a las funciones que le fueran encomendadas y que sintetizan en dos dimensiones de trabajo relacionadas entre sí. Por un lado, la función de favorecer y articular las prácticas integrales universitarias en territorio, incluyendo su monitoreo y evaluación participativos. Por otro lado, el desarrollo de las tres funciones universitarias de investigación, extensión y enseñanza, desde un enfoque territorial. De este modo, la organización en núcleos buscó articular las líneas estratégicas o elementos priorizados de base territorial, de forma de integrar la intervención con la generación de conocimiento y propiciar la inserción estratégica de los servicios universitarios. Así, los Núcleos de Intervención e Investigación son espacios relevantes en tanto piensan las prácticas que desarrolla el Programa a partir de campos temáticos delimitados sobre la base de las referencias conceptuales y metodológicas que surgen de nuestra praxis. Estos también albergan las referencias territoriales del Programa (vínculos con territorios y actores que se tienen y se tendrán) y los formatos/dispositivos de trabajo concretos en los que se participa.

Ahora bien, ¿qué implica el enfoque territorial? Entendemos el territorio como una construcción social e histórica sobre el espacio que es al mismo tiempo producto y productor de los sujetos que lo habitan, lo que implica siempre una cierta apropiación. Los geógrafos Roger Brunet, Robert Ferras y HervéThéry (1993) sostienen que la noción de territorio es a la vez jurídica, social, cultural, pero también afectiva. En ese sentido, el concepto de territorio va de la mano de la construcción de múltiples territorialidades que operan dentro del mismo y generan relaciones de complementación y cooperación pero también de conflicto y disputas. Por tanto, como afirma Lopes de Souza (1995), el territorio es "el espacio definido y delimitado por y a partir de relaciones de poder", esto es, "un campo de fuerzas, una tela o una red de relaciones sociales que, a la par de su complejidad interna, define al mismo tiempo un límite, una alteridad: la diferencia entre 'nosotros' (un grupo, los miembros de una comunidad) y los 'otros' (los de afuera, los extraños)" (p. 86). En esta misma línea, el concepto de "territorialidad" remite a una construcción cultural del espacio y se asocia con apropiación y con identidad y afectividad espacial, que se combinan para definir "territorios apropiados de derecho, de hecho y afectivamente" (Montañez Gómez; Delgado Mahecha,1998:1-2).

Esta forma de concebir y problematizar el territorio donde el Programa lleva adelante sus actividades visibilizando las territorialidades que se complementan o se oponen, los procesos de identificación, las prácticas y experiencias de los sujetos que transitan y las relaciones de poder que conviven, nos condujo al armado de diferentes Núcleos de Intervención e Investigación. Asi, esos tres Núcleos consolidados recogen las producciones acumuladas en torno a los campos temáticos sobre los que el Programa viene avanzando en el último tiempo para su vinculación universidad-sociedad a nivel territorial, y son los siguientes: i) Territorio, Comunidad y Derechos Colectivos; ii) Educación y Territorio; iii) Mundo del Trabajo, Ambiente y Organizaciones Sociales.

De este modo, se busca hacer operativa la noción de integralidad, entendida como una clave para la renovación de la enseñanza, la incorporación de la extensión al curriculum y la aproximación de la investigación a esas dos funciones universitarias que define a la extensión como función con capacidad de redimensionar a las demás (a los procesos de aprendizaje, la enseñanza y la investigación). Es posible identificar en el proceso de cons- 
trucción de una visión integral de la realidad algunas dimensiones claves que interactúan con la concepción y práctica de la educación popular, considerada como un movimiento de búsqueda de construcción de nuevos saberes y de una nueva cultura desde una intencionalidad transformadora. Estas prácticas conforman un cuerpo teórico y metodológico que trasciende fronteras disciplinares y potencia el aporte de cada disciplina en el conocimiento articulado de la realidad.

\section{Características socioeducativas en el espacio geográfico de incidencia del PIM}

El espacio territorial de inserción del PIM comprende los Municipios F y E del departamento de Montevideo y el Municipio de Barros Blancos del departamento de Canelones (ver Figura 1), un área que abarca unos $110,8 \mathrm{~km}^{2}$ y que para el año 2011 tenía 259.613 habitantes.

Figura 1. Territorio de referencia del Programa Integral Metropolitano.

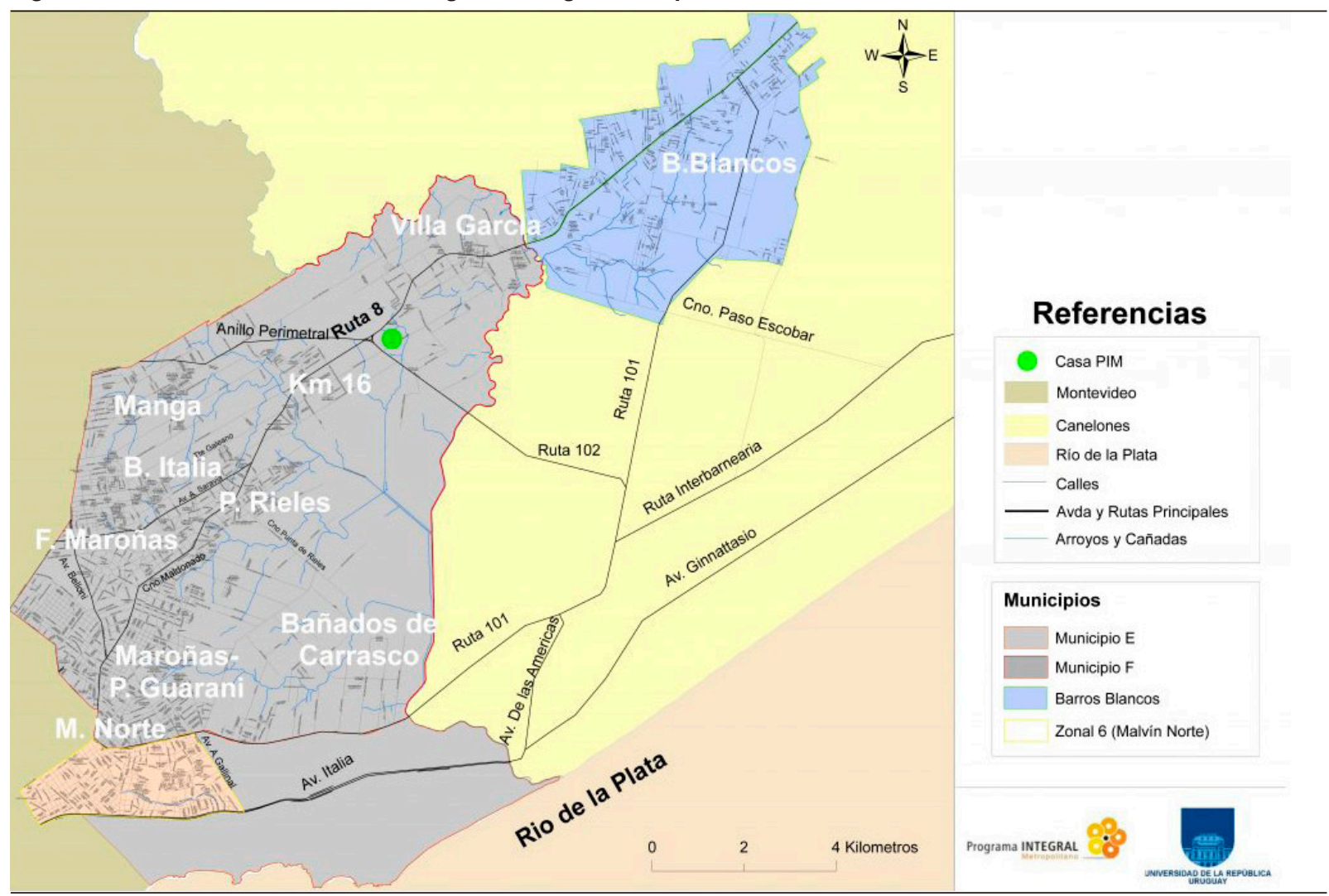

Fuente: PIM UdelaR, 2019

Estas zonas son sumamente heterogéneas; mientras que la superficie que abarca el Municipio E (donde habitaban 153.395 personas en 2011) se caracteriza por ser netamente urbana y la del Municipio $F$ (donde habitaban 168.910 personas) comprende áreas urbanas y rurales, la del Municipio de Barros Blancos (en donde habitaban 27.687 personas) conforma 
una microrregión de Canelones de vocación industrial tecnológica - junto con las localidades de Suárez, Empalme Olmos, Toledo y Pando (INE, 2011).

Una de las principales características de este territorio (que comparte con el resto de la zona noreste de Montevideo) es la presencia y convivencia de espacios rurales y urbanos así como un fuerte proceso de metropolización (ROU, 2007 y Veiga, 2009) del área rural a partir de la expansión urbana residencial e industrial y de servicios. Este proceso trajo aparejada una serie de fenómenos como son la fuerte desaparición de productores, la pérdida de áreas de suelo dedicado a la actividad agropecuaria, el deterioro del suelo e importantes superficies improductivas.

En ese sentido, estas transformaciones territoriales del área metropolitana han desencadenado fuertes procesos de segregación socioespacial y fragmentación urbana (ROU, 2007) que transformaron la diversidad campo-ciudad en desigualdad social: la población en condición de pobreza fue concentrándose en territorios diferentes de aquello en los que se fueron localizando las clases medias; se consolidaron inequidades en el acceso al transporte público y el tejido urbano fue perdiendo heterogeneidad social en un proceso de fragmentación y diversificación de los dos extremos de la población urbana y la estructura social (INEEd, 2014) que refleja la influencia de los procesos de reestructuración económica, los cambios en el mundo del trabajo, la nueva composición de los hogares y roles familiares y la diversificación de pautas culturales y de consumo (Veiga, 2009:148).

La situación social crítica por la que han pasado y que aún prevalece en las distintas subáreas o localidades compromete a gran parte de la población del área metropolitana, en donde se encuentran 80 asentamientos irregulares, 65 de los cuales (para el año 2010) se ubicaban en el Municipio F. Los problemas estructurales que se arrastran desde hace décadas tienen que ver con los aspectos productivos (falta de fuentes de trabajo, migración campo ciudad, etc.) y se relacionan también con la ausencia de políticas públicas de desarrollo adecuadas (infraestructura urbana deficiente, transporte, ausencia y/o deficiencia de los servicios sociales básicos, etcétera).

Desde el punto de vista de la dinámica poblacional, en el período intercensal 1996-2004, en estos territorios también es posible advertir un proceso de enlentecimiento en el aumento de la población de los sectores socioeconómicos medios y altos y un aumento de la población en aquellos sectores socioeconómicos más bajos, los cuales también se componen mayoritariamente por población femenina y en los primeros tramos de edad.

En cuanto a los datos educativos, el censo 2011 muestra una tasa neta de asistencia de 6 a 11 años en educación escolar del 91,4\%, levemente más baja que el total del país $(92,9 \%)$. Sin embargo, si miramos la tasa neta de asistencia de 12 a 17 años en educación media, la brecha crece, es de $60,7 \%$ frente a $67,7 \%$ del total del país. El municipio $\mathrm{F}$, en comparación con otras zonas de la ciudad, presenta un cierto déficit en la oferta de centros de enseñanza secundaria, lo cual es un factor más que afecta la desvinculación temprana del sistema educativo de los adolescentes. Además, los datos educativos que disponemos del censo 2011, con relación al máximo nivel educativo alcanzado por la población de 25 años y más, muestra un porcentaje mayor que el total país para la educación primaria y ciclo básico, pero cambia rotundamente si miramos los datos de bachillerato o educación terciaria y universitaria, en donde los porcentajes son mucho menores que el total del país. 
Estos datos cuantitativos presentes en el territorio nos sugieren que en el espacio geográfico en el que se desenvuelve el Programa se conjuga una multiplicidad de territorios y territorialidades que condicionan posicionamientos sociales, identidades, prácticas cotidianas y proyectos laborales diferenciados que construyen subjetividades diversas. Asimismo, nos ayuda a pensar al territorio como una articulación, compleja y dinámica, de factores históricos, económicos, sociales, políticos y culturales, sobre los que un programa territorial como el PIM debe indagar, visibilizar y construir alternativas posibles de inserción.

\section{Pensar lo pedagógico en el territorio: Núcleo de Intervención e Investigación Educación y Territorio}

Como fue dicho, estos Núcleos tienen por cometido articular la investigación interdisciplinaria en torno a las problemáticas emergentes del territorio, favorecer su conocimiento complejo y nutrir las líneas de extensión y enseñanza en una perspectiva integral. Para ello, cada núcleo genera acuerdos de trabajo con equipos académicos de diferentes áreas de conocimiento de la UdelaR y conforma equipos de investigación y extensión más amplios a través de los dispositivos existentes en la Universidad: llamados a proyectos, acuerdos de trabajo, entre otros mecanismos.

Tal como plantea Adriana Puiggrós (1990), la educación no es una "unidad abstracta" sino un "campo problemático", cuya

"relación con otros campos no seguiría criterios de búsqueda de una unidad actual esencial de lo social ni la creencia de la última posibilidad de continuidad absoluta de los campos problemáticos, sino la aceptación de la ruptura, discontinuidad e imposibilidad de suturación final entre la educación, la política, la sociedad, criterio que es condición para la interdisciplina". (1990:10)

Se trata de una formulación de la educación como problema apropiada para su abordaje desde el PIM que incluye una diversidad de ámbitos y niveles.

De este modo, el Núcleo de Intervención e Investigación Educación y Territorio tiene como objetivo conocer las características y problemáticas educativas de las zonas de referencia del PIM. A su vez, busca sistematizar y aportar al diálogo de saberes y a la discusión colectiva en torno al tema de las alternativas pedagógicas y las alteraciones de la forma escolar en los procesos educativos realizados en escuelas, liceos, comunidades, organizaciones e instituciones de los barrios contemplados en el Programa. Comprende procesos pedagógicos desarrollados tanto dentro como fuera de las instituciones escolares, el estudio de la propia producción de esas relaciones de frontera-integración adentro-afuera de las instituciones, los procesos de educación popular realizados de modo autónomo por colectivos y organizaciones, y la reflexión pedagógica respecto de los propios procesos formativos y extensionistas universitarios. Todo ello constituye el universo del "campo problemático" de la educación para el PIM.

A partir del trabajo en el territorio, del análisis de la coyuntura político-educativa y social, así como de las opciones teórico-metodológicas que el equipo efectuó, se priorizaron algunas líneas de investigación en cuanto a temáticas y problemáticas emergentes transversales a los diferentes procesos de extensión y articulación con las instituciones educativas y organi- 
zaciones. Su formulación y concepción procuran convocar a la participación interdisciplinaria. A continuación, presentamos las cuatro líneas de investigación e intervención del Núcleo.

\section{a) La educación y las construcciones de lo común}

La educación, en tanto proyecto cultural organizado desde el Estado, y como construcción ciudadana en diferentes niveles, está intrínsecamente ligada, al menos desde la Modernidad, al intento de construir sociedades culturalmente homogéneas a partir de su normalización por medio de la transmisión intergeneracional de un legado cultural y una identidad histórica. Por cierto, cualquier intento de homogeneizar lo social se convierte en un fallido, en tanto "la sociedad es imposible", tal como señalan Laclau y Mouffe (2011) al evidenciar el carácter constitutivo de lo social que tiene el conflicto, ya que la idea de integración social plena se ve socavada por el conjunto de desigualdades y diferencias (de clase, de género, entre otras) que caracterizan a las sociedades del capitalismo globalizado. Así las cosas, la referencia a "lo común" despliega un campo de problemas relacionados con el papel de la educación en la dinámica de producción de lo social, respecto de concepciones y políticas que articulan o tensionan al campo pedagógico con referencia a los polos justicia-injusticia e igualdad-desigualdad. Tanto más en territorios como el del PIM, que están caracterizados por situaciones de segregación social y territorial con alto impacto en lo educativo.

La noción de «lo común» o «los comunes» ha tenido un importante desarrollo en el tiempo reciente, tanto por parte de autores europeos, como Laval y Dardot (2014) y Garcés (2013), como por latinoamericanos como Gago (2015), Zibechi (2010), Pinheiro (2016), además de producciones de movimientos sociales como el zapatismo en México. Por su parte, Cornú nos dice que

"sentir al otro como mi semejante, decirlo, reconocerlo (...), es imaginarse en su lugar, imaginarse en ese otro lugar; es tener la idea de los puntos de vistas diferentes, pero no incompatibles (...) [y en ese sentido, la cuestión de lo común en educación sería] cómo pensar el actuar con hospitalidad con relación a quien siempre está por llegar. (...) la experiencia en común tiene una dimensión sensible que constituye la metáfora de una comunidad por venir, jamás cerrada" (2012:142-144).

En esta línea de investigación, que reflexiona sobre cómo se construye lo común desde experiencias pedagógicas diversas, integramos una indagación específica sobre "Educación, Interculturalidad y Migraciones". Al respecto, en los últimos años, Uruguay ha manifestado cambios importantes en las características de su flujo inmigratorio que no tienen que ver solo con el crecimiento en su volumen total sino también con la composición por orígenes, donde la mayor novedad es el crecimiento del porcentaje de personas provenientes de orígenes latinoamericanos de países no limítrofes, como Cuba, Venezuela y República Dominicana. Así, el análisis de los espacios pedagógicos en los que se despliegan dichos colectivos nos permite interpelarnos sobre la construcción de múltiples pertenencias e identidades y sobre el lugar que adquiere la escuela en los procesos de una integración intercultural. También contribuye a pensar en las problemáticas de la diversidad cultural, la integración social y la construcción de lo "común" en espacios sociales con claras desigualdades y opresiones de diferente tipo que atraviesan el acto pedagógico en el territorio. $Y$ nos interpela sobre las formas diversas de socialización y saberes que convergen en la escuela y en otros espacios 
de aprendizaje no escolarizados para reflexionar sobre diferentes aspectos que definen la identidad y la ponen en juego en los territorios educativos, como la ascendencia étnico-racial, los mandatos de género y la interculturalidad, entre otros. De esta forma, concebir lo educativo desde un territorio específico y con sujetos sociales diversos, situando lo "diverso" y lo "común" como dos caras de una misma moneda que convergen en el acto pedagógico y visibilizando las relaciones de poder que reproducen las desigualdades, nos permite pensar en un proyecto político-epistemológico y educativo que busque el reconocimiento de los saberes de la comunidad y el establecimiento de relaciones simétricas entre las personas de distintos género, culturas y condición social.

\section{b) Alternativas pedagógicas y sistematización de experiencias}

Nuestra intención es conocer, sistematizar, describir e interpretar experiencias educativas transformadoras realizadas por educadores/as en el territorio del PIM. Una construcción teórico-metodológica dirigida a indagar las experiencias alternativas dentro del campo problemático de la educación fue la del Programa Alternativas Pedagógicas y Prospectiva Educativa en América Latina (APPEAL) conformado por investigadores de la Universidad de Buenos Aires (UBA) y la Universidad Nacional Autónoma de México (UNAM) y con equipos de investigación en otros países del continente. Dicho Programa contituyó un conjunto abierto de referentes teóricos, articulados en una propuesta metodológica de sistematización de experiencias que posibilitaron abordar el estudio de lo educativo, a la vez, en su autonomía relativa y su relación con los procesos sociales históricos que lo sobredeterminan (Gómez y Corenstein, 2014). Así, lo hegemónico y lo alternativo no son estados puros que se alcanzan sino lógicas que se tensan y disputan con fuerza desigual en la producción de contenidos, formas, sentidos y significados relacionados con lo educativo. No se trata de considerar lo alternativo como portador de una virtud intrínseca o a priori, como propaga cierto discurso de mercadeo educativo. De lo que se trata es de examinar los sentidos y contenidos asignados a las construcciones alternativas, incluyendo la rearticulación de elementos propios de las tradiciones hegemónicas.

Nuestro enfoque teórico-metodológico procura articular los aportes de APPEAL con los de la línea de investigación sobre "alteración de la forma escolar" (Frigerio y Diker, 2010), en particular con los desarrollos que en nuestro medio realizó el Grupo de Estudios en Políticas y Prácticas Educativas de Facultad de Humanidades y Ciencias de la Educación (GEPPrEd) (Martinis, 2016; Stevenazzi, 2014). Como estrategia metodológica, hemos combinado la matriz de sistematización de APPEAL con instrumentos de la Investigación Acción Participativa, integrando a los/as educadores como coinvestigadores.

El trabajo de sistematización de experiencias alternativas abarca tanto experiencias presentes como una línea específica de "memorias pedagógicas". Y uno de los referentes empíricos para trabajar esta línea es el trabajo territorial que se viene llevando a cabo en Villa García (barrio de Montevideo), en donde indagamos en la construcción de memorias barriales y la categoría de memorias pedagógicas. Al respecto, durante la década de 1950 y en 1975, se desarrolló una experiencia educativa en la zona de Villa García que, a la vez que no puede ser disociada del nombre del director de la Escuela Rural No 157 durante ese período -Mtro. José Pedro Martínez Mattonte-, tampoco puede serlo de la multiplicidad de personas que conformaron lo que se denominó Unidad Educacional Cooperaria (UEC) de Villa García. 
Esta experiencia fue fruto de las acciones de cooperación entre la Dirección de la Escuela, maestras, estudiantes, familias, instituciones educativas y organizaciones comunitarias, y produjo un aporte significativo a la vida cultural de la Villa: Escuela Rural, Liceo Popular, Talleres de Oficios, Policlínica, Alojamiento Estudiantil, Comedor, Huerta, Granja, Asambleas. El objetivo de este abordaje es, por un lado, recuperar, historizar y problematizar la experiencia de la UEC y la labor del Maestro Martínez Matonte como una alternativa pedagógica muy particular, que pudo ser posible por la conjunción de factores culturales, sociales, políticos y territoriales precisos.

Asimismo, se busca profundizar la conceptualización de la categoría de memorias pedagógicas, cuanto a indagar en la vigencia del legado pedagógico y social de la UEC, en las experiencias actuales de la Escuela $N^{\circ} 157$, el Liceo $N^{\circ} 52$, el Jardín $N^{\circ} 348$, la Coordinadora Vecinal y la comunidad de Villa García. En ese sentido, consideramos que la memoria individual y colectiva cobra un valor simbólico que revela aspectos significativos acerca de cómo se piensa el colectivo. La reconstrucción de ese pasado en el presente de la comunidad educativa de Villa García implica una operación política sobre la cual se construye una identidad cultural y pedagógica particular (Paleso y Moreira, 2019).

\section{c) Prácticas universitarias en territorio}

Como docentes de un Programa Integral de abordaje territorial realizamos una gran variedad de prácticas educativas. La enseñanza integral supone una práctica docente en construcción (Cano y Castro, 2013; Castro, 2015; Cavalli, 2019) en el marco de un modelo educativo que favorece la formación y se reconfigura y transforma (Cano y Castro, 2013; Castro, 2015; Fraga, 2017; Cavalli, 2019). Los sujetos no universitarios y sus saberes constituyen la escena educativa y sus características (Facchinetti, 2014). Nos enfrentemos a prácticas educativas en las que se redoblan las diversidades: de sujetos, de territorios, de saberes, de temas, de objetivos de formación, y que se desarrollan en el marco de propuestas que propician una instancia de saber (Behares, 2011), en un proceso que apuesta al movimiento y la multiplicidad, que favorece encuentros colectivos y la conformación de lo común.

Decimos, entonces, que nuestras prácticas son a la vez específicas y diversas. Específicas en tanto suponen un modo distinto de hacer docencia, puesto que se articulan funciones, saberes y disciplinas hacia la búsqueda de algo nuevo, nuevos pensamientos y saberes. Diversas porque en cada propuesta, en cada EFI, estas prácticas, las modalidades de enseñanza y las herramientas didácticas que en ellas se despliegan dependen de la especificidad del tema, de los sujetos con quienes se desarrollan. Son prácticas situadas y constituidas en territorio.

Nos encontramos ante una enseñanza que se renueva y se transforma. Un curso nunca es el mismo en sus diferentes ediciones; un EFI nunca es el mismo. Es por ello que vemos en nuestras propuestas educativas una oportunidad para investigar y producir conocimiento.

Asimismo, identificamos sublíneas de investigación específicas que componen el problema de las prácticas universitarias en territorio:

- Especificidad y diversidad en de las prácticas docentes integrales.

- Monitoreo, evaluación y autoevaluación de las prácticas integrales en territorio y del PIM.

De esta manera, visualizamos en nuestras propuestas educativas una oportunidad para investigar y producir conocimiento acerca de la docencia integral y las modalidades y estra- 
tegias didácticas que en ellas se desarrollan. Entendemos que el análisis de nuestras propias prácticas debe ser constante, apostar a un ejercicio de reflexividad como proceso de aprendizaje y transformación. Debe identificar aquellas actividades, modalidades, estrategias que nos resultan interesantes, novedosas, útiles, pero también aquellas que obstaculizan o dificultan el desarrollo de una propuesta integral y sus objetivos.

A su vez, la existencia de diversas intencionalidades y direccionalidades en las experiencias de prácticas integrales universitarias evidencia la necesidad de sistematizar y estudiar las metodologías utilizadas para su desarrollo, de forma de permitir mejorar el vínculo entre el ejercicio docente integral, las necesidades de los estudiantes y de transformación de situaciones sociales (Acosta y Bianchi, 2015).

La reflexión colectiva y el desarrollo de estrategias de monitoreo y evaluación adecuadas a las prácticas integrales universitarias que se desarrollan en el PIM resultan no solo necesarios sino de gran valor para el análisis y perfeccionamiento del equipo docente en las actividades desplegadas. Complementariamente, el diseño de criterios e indicadores pertinentes para la autoevaluación institucional resulta de gran valor tanto para la toma de decisiones informadas en las actividades del programa académico como para el aprendizaje colectivo de los actores vinculados a las prácticas del PIM.

\section{d) Saberes, aprendizajes y experiencias con relación al trabajo}

El trabajo, desde la perspectiva de la integralidad, orientado por una actividad extensionista, supone el vínculo con colectivos, organizaciones, instituciones, vecinas y vecinos no organizados, entre otros. En las prácticas cotidianas que estos desarrollan se despliegan diferentes saberes (Cavalli, 2019; Deleuze, 2013; Foucault, 2013; Charlot, 2000) con los cuales apostamos a generar prácticas desde el diálogo de los mismos. En estas prácticas son puestas en juego las maneras de adquirir y transmitir estos saberes y se determina también cómo circulan y se relacionan con otros. Así, entendemos que las experiencias laborales implican un espacio significativo para la producción de saberes socialmente productivos (Puiggrós, 2004).

Encontramos una nueva línea de investigación en la cual nos preguntamos sobre los saberes de los actores no universitarios con los que trabajamos pero igualmente sobre los modos que estos se dan para aprender y enseñar estos saberes. En primer término, nos proponemos analizar esta problemática en los colectivos que se componen como espacios de trabajo (formal y no formal, remunerado o no remunerado), ya que consideramos que mucha de su actividad se da en torno a saberes específicos.

Nos preguntamos acerca de la comprensión que los sujetos tienen de sus propios saberes así como del reconocimiento de los mismos como saberes o conocimientos específicos y valiosos. Por otro lado, nos interesa identificar los modos y estrategias que desarrollan para adquirir, transmitir y producir saberes, y si los sujetos asimilan que se realizan con dicho fin.

A su vez, consideramos relevante explorar la forma en que circulan y se relacionan estos saberes dentro del colectivo, entre diferentes colectivos y con el resto de la comunidad.

En esta línea, se conformó un proyecto de investigación titulado "Saberes y trabajo. Generación, aprendizajes y circulación de saberes en experiencias laborales de la región metropolitana de Montevideo", el cual es llevado adelante en conjunto con el núcleo Mundo del Trabajo, Ambiente y Organizaciones Sociales, lo que permite nutrir el campo-tema (Spink, 2005) a abordar desde la mirada del trabajo y la educación. 


\section{Comentarios finales}

En nuestro enfoque buscamos enfrentar la fragmentación y segregación del sistema educativo público, pasando de una diferenciación indiferente de sus subsistemas a una complementación comprometida. Los problemas educativos de una parte del sistema deben concebirse como problemas de la educación pública en su conjunto, lo que permite que sean los diferentes subsistemas los que se comprometan también en su estudio y abordaje, poniendo sus diferencias de roles y cometidos al servicio de dichos abordajes y ya no como justificación de una indiferencia y una fragmentación disfrazadas de especialización. Esto es más sencillo de enunciar que de hacer. Las dificultades provienen de la complejidad y dimensión del sistema educativo y de las diferentes tradiciones que en su proceso histórico se han conformado, sumadas al factor subjetivo de que educadores, estudiantes y militantes de la educación somos en parte esas tradiciones institucionales; nuestra visión de los temas educativos tiene allí su matriz y de allí parten muchos de nuestros desencuentros ocasionales. En este marco, desde el PIM procuramos aportar a nuevas articulaciones entre los subsistemas, y para ello partimos de lo territorial, lo zonal, lo regional, de manera de crear y llevar a cabo propuestas educativas conjuntas así como proyectos de investigación y formación permanente.

Las líneas de investigación se articulan con procesos de extensión junto a instituciones educativas y actores del territorio, y a su vez nutren un curso de grado que es abierto tanto a estudiantes de la UdelaR como de Formación Docente. Dicho curso tiene en 2019 su segunda edición con el nombre "Pedagogía política y territorio", es coorganizado entre el PIM y el Grupo de Estudios en Políticas y Prácticas Educativas de la Facultad de Humanidades y Ciencias de la Educación de la UdelaR. Estructurado como EFI, posee una duración anual y una propuesta formativa de carácter teórico-práctico. Los/as estudiantes realizan experiencias de inserción en algunos de los procesos de extensión y/o investigación del PIM junto a instituciones educativas y sus comunidades.

Estas experiencias tienen características diferentes pero, como elementos comunes, procuran construir un proyecto educativo anual que disponga a dialogar a los centros educativos con su entorno barrial, articulando las asignaturas entre sí y los/as profesores/as y estudiantes del Liceo con sus pares de la Universidad. Esta propuesta es construida con los/ as educadores/as del Liceo y se basa en los contenidos curriculares de sus programas, de modo tal que se favorezca una perspectiva pedagógica en la que el docente dé continuidad a su rol de educador extendiendo el aula a nuevas situaciones y formatos.

La ejecución de esta perspectiva de trabajo encuentra obstáculos de diferente naturaleza que, juntos, conforman una trama que impide dar un salto cualitativo en su desarrollo, consolidación y expansión. La sistematización adecuada de estos obstáculos está aún por realizarse, tarea necesaria para poder nutrir transformaciones a nivel de la política educativa y curricular tanto en la Universidad como en Enseñanza Media. A cuenta de un análisis específico sobre esta trama de dificultades, que no es el objeto de este artículo, simplemente daremos algunos ejemplos de los elementos que atentan contra este tipo de experiencias. Por una parte, existen dificultades en la naturaleza diferente de los contratos docentes a nivel de la Universidad y de Enseñanza Media. Mientras que, en el primer caso, efectuar proyectos de extensión e investigación junto a los liceos e instituciones presentes en el territorio es parte de nuestra tarea, prevista y remunerada, para el caso de los/as profesores/as de media 
esto no es así, ya que no cuentan con horas previstas para coordinar y desarrollar este tipo de proyectos. Esta situación les lleva a hacer verdaderas proezas administrativas para poder dedicarse a estas actividades, que siempre conllevan un tiempo mayor del que tienen contratado. A lo que se agrega una pesadez burocrática desgastante. Este cúmulo fortalece una dinámica de tipo voluntarista que atenta contra la proyección en el tiempo de los proyectos.

Otros problemas que hemos encontrado refieren a las distintas estructuras curriculares (semestrales en el caso de la Universidad, anual en el caso de Enseñanza Media), a la dinámica de los proyectos concursables con que se desarrolla gran parte de la actividad de extensión en la Universidad, entre otros aspectos.

Con todo, una nueva configuración estructural y curricular del sistema de educación pública que favorezca las articulaciones entre instituciones y niveles tendrá una buena base donde apoyarse si desde las estructuras actualmente existentes se ensayan proyectos conjuntos desde las realidades locales y las necesidades del territorio. Nuestra experiencia junto a las instituciones educativas de la zona del PIM nos refuerza el valor de estas iniciativas y su potencial de desarrollo. Divulgar e intercambiar sobre estas líneas de trabajo, pensamos, puede contribuir a aportar otras líneas de reflexión para un debate educativo que, en la actualidad, con frecuencia queda clausurado por lógicas productivistas, tecnocráticas y econométricas dominantes. En esa dirección, en el presente artículo hemos procurado compartir una perspectiva teórica, metodológica, política y organizativa para abordar el "campo problemático" de la educación desde la implicación de la Universidad con las problemáticas que aborda desde el territorio, sin perder la especificidad de aporte universitario de construir conocimiento crítico desde pero también más allá del territorio.

\section{Referencias bibliográficas}

Behares, L. (2011). Enseñanza y producción de conocimiento. La noción de enseñanza en las políticas universitarias uruguayas. Montevideo: Departamento de Publicaciones de la Universidad de la República.

Brunet, R.; Ferras, R. y Thery, H. (1993). Les Mots de la Géographie, dictionnaire critique. Paris: Reclus-La documentation française.

Cano, A (2015). Integralidad en la Universidad de la República. Apuntes para el debate universitario. En En clave inter 2014. Educación superior e interdisciplina (pp. 11-18). Montevideo: Espacio Interdisciplinario de la Universidad de la República.

Cano, A. y Castro, D. (2013). Análisis de los modelos pedagógicos en las prácticas educativas universitarias que integran la extensión a la formación curricular. Informe Final de Investigación, Inédito. Instituto de Perfeccionamiento y Estudios Superiores, ANEP. Montevideo.

Cavalli, V. (2019). Imágenes de un aula en movimiento. Pensamientos sobre los saberes en la enseñanza universitaria integral. Tesis de Maestría no publicada. UdelaR, Montevideo.

Charlot, B. (2000). La relación con el saber. Elementos para una teoría. Montevideo: Trilce.

Cornú, L. (2012). Lugares y formas de lo común. En Frigerio, G. y Dikers, G. (Comps.). Educar: posiciones acerca de lo común. Buenos Aires: Del Estante.

Deleuze, G. (2013). El saber. Curso sobre Foucault. Buenos Aires: Cactus.

Foucault, M. (2013). La arqueología del saber. Buenos Aires: Siglo XXI.

Fraga, S (2017). En clave de integralidad: aprender-haciendo desde las prácticas docentes de enseñanza en extensión universitaria. Tesis de Maestría no publicada. UdelaR, Montevideo. 
Frigerio, G. y Diker, G. (Comps.) (2010). Educar; saberes alterados. Buenos Aires: Del Estante.

Gago, V. (2015). La razón neoliberal. Economías barrocas y pragmática popular. Buenos Aires: Tinta Limón.

Garcés, M. (2013). Un mundo común. Barcelona: Bellaterra.

Gómez, M. y Corenstein, M. (2014). Reconfiguración de lo educativo en América Latina. Experiencias pedagógicas alternativas. México DF: UNAM.

INEEd (2014). Informe sobre el estado de la educación en Uruguay 2014. Montevideo: INEEd.

Laval, C. y Dardot, P. (2014). Común. Ensayo sobre la revolución en el siglo XXI. Barcelona: Gedisa.

Laclau, E. y Mouffe, C. (2011). Hegemonía y estrategia socialista. Buenos Aires: Fondo de Cultura Económica. Lago, G.; Rimbaud, T. \& Venegas, M. (2017). Extensión, investigación e integralidad en la Facultad de Arquitectura, Diseño y Urbanismo de la Universidad de la República. +E: Revista de Extensión Universitaria, 6(6), 154-159. Santa Fe, Argentina: Ediciones UNL. doi: https://doi.org/10.14409/extension.v1i6.6324

Lopes de Souza, M. (1995). O territorio. Sobre espaço e poder, autonomia e desenvolvimento. En Castro, I.E.; Da Costa Gomes, P.C. y Lobato Correa, R. (Orgs.). Geografia, conceitos e temas (pp. 77-116). Rio de Janeiro: Bertrand.

Martinis, P. (2016). Sujeto de la educación y discurso pedagógico (social) O acerca de la no continuidad entre sujetos sociales y sujetos educativos. En Camors, J. Reflexiones sobre las prácticas educativas en Brasil y Uruguay Pedagogía social y educación social (pp. 39-52). Montevideo: FHCE.

Montañez Gómez, G.; Delgado Mahecha, O. (1998). Espacio, territorio y región: conceptos básicos para un proyecto nacional. Cuadernos de Geografía - Revista del Depto. de Geografía, 1-2.

Núñez, L.; Américo, F., Arias, J. y Thevenet, G. (2017). Experiencias de formación en extensión universitaria. Aportes y desafíos institucionales. +E: Revista de Extensión Universitaria, 7(7), 234-243. Santa Fe, Argentina: Ediciones UNL.

Stevenazzi, F. (2014). Una lectura sobre la producción de alteraciones a los formatos escolares desde la política cotidiana de la escuela. Vozes dos Vales, III(6). UFVJM.

Paleso, A. y Moreira, C. (2019). La Unidad Educacional Cooperaria en la trama de la memoria: prácticas pedagógicas del pasado puestas en el porvenir. Hemisferio Izquierdo, (31). Montevideo.

Pinheiro, L. (2016). Educación, resistencia y movimientos sociales: la praxis educativo-política de los Sin Tierra y de los Zapatistas. México: UNAM.

Programa Integral Metropolitano (PIM) (2008). De Formaciones in-disciplinadas. Montevideo: UdelaR.

Puiggrós, A. (1990). Imaginación y crisis en la educación latinoamericana. México DF: Alianza Editorial Mexicana. (2004). El lugar del saber. Buenos Aires: Galerna.

Rinesi, E. (2015). Filosofía (y) política de la Universidad. Buenos Aires: UNGS.

ROU (2007). Libro blanco del área metropolitana (Canelones, Montevideo, San José). Presidencia de la República, Uruguay: Tradinco.

Spink, P. (2005). Replanteando la investigación de campo: relatos y lugares. Athenea Digital, 8. doi: https:// doi.org/10.5565/rev/athenead/v1n8.238

Tommasino, H., Cano, A., Castro, D. Santos, C. y Stevenazzi, F. (2010). De la extensión a las prácticas integrales. En: Hacia la Reforma Universitaria. La extensión en la renovación de la enseñanza: los Espacios de Formación Integral. Montevideo: Rectorado de la UdelaR.

Tommasino, H., \& Stevenazzi, F. (2017). Reflexiones en torno a las prácticas integrales en la Universidad de la República. +E: Revista de Extensión Universitaria, 6(6), 120-129. https://doi.org/10.14409/extension.v1i6.6320

UdelaR (2010). La extensión en la transformación de la enseñanza: Ios Espacios de Formación Integral. Hacia la Reforma Universitaria, (10). Montevideo: SCEAM.

— (2019). "¿Qué es el PIM?”. Recuperado http://pim.udelar.edu.uy/institucional/que-es-pim/ (11/06/19) 
Veiga, D. (2009). Desigualdades sociales y fragmentación territorial en el área metropolitana de Montevideo. Lider, 15(11).

Zibechi, R. (2010). Movimientos y emancipaciones. Del desborde obrero de los '60 al "combate a la pobreza". Montevideo: Alter Ediciones. 